\title{
AN $A$-PROPER MAP WITH PRESCRIBED TOPOLOGICAL DEGREE
}

\author{
MALCOLM COUSLAND ${ }^{1}$
}

\begin{abstract}
For any given element $\alpha$ of the ring ${ }^{*} Z=Z^{N} / I$, where $I$ is the ideal of integer sequences convergent to 0 , an $A$-proper map in $l_{2}$ is constructed whose degree in the sense of S. F. Wong is equal to $\alpha$.
\end{abstract}

The concept of an $A$-proper map acting in a Banach space was introduced by Petryshyn [6, p. 157] (originally called an operator satisfying condition (H)). A topological degree for such maps was defined by Browder and Petryshyn [1], [2] as a generalization of the Leray-Schauder degree [5] for maps of the form Identity + Compact. Browder and Petryshyn established the basic properties of their degree, Deg $(T, G, y)$, which is set valued (see the note after Definition 3 for its definition), invariant under suitable homotopies, satisfies a weakened sum formula

$$
\operatorname{Deg}(T, G, y) \subseteq \operatorname{Deg}\left(T, G_{1}, y\right)+\operatorname{Deg}\left(T, G_{2}, y\right),
$$

and if $\operatorname{Deg}(T, G, y) \neq\{0\}$ then there is an $x \in G$ such that $T(x)=y$. Wong [9] has given a new definition of the degree with values in a ring ${ }^{*} Z$ (see below) which satisfies the sum formula with an equality sign. Later Wong [10] proved a restricted product formula for the degree of the product $T U$ under the restriction that at least one of the maps $T$ or $U$ must be of the form Identity + Compact.

The purpose of this paper is, given $\alpha \in{ }^{*} Z$, to construct an $A$-proper map in $l_{2}$ whose degree is $\alpha$. In the following let $X$ be a real Banach space. Let $\operatorname{cl}(G)$ denote the closure of $G$ and $\partial G$ the topological boundary of $G$ for subsets $G$ of $X$.

Definition 1. An (oriented) projectionally complete scheme $\Gamma$ for mappings from subsets of $X$ to $X$ is a monotonically increasing sequence $\left\{X_{n}\right\}$ of (oriented) finite dimensional subspaces of $X$ and a sequence $\left\{P_{n}\right\}$ of continuous linear projections $P_{n}: X \rightarrow X_{n}$ with $P_{n} X=X_{n}$, such that $P_{n} x \rightarrow x$ as $n \rightarrow \infty$ for each $x \in X$.

This definition is adapted from Fitzpatrick [4, Definition 1.1, p. 537]. The following definition is that of Petryshyn [7, Definition 1, p. 271].

Definition 2. Let $G$ be a subset of $X$, and $\Gamma$ a projectionally complete

Received by the editors May 27, 1975.

AMS (MOS) subject classifications (1970). Primary 47H99; Secondary 55C25.

Key words and phrases. A-proper map, topological degree, projectionally complete scheme, oriented projectionally complete scheme.

1 The author's research was supported by a Commonwealth Postgraduate Research Award. 
scheme in the sense of Definition 1. The mapping $T: \operatorname{cl}(G) \rightarrow X$ is $A$-proper with respect to $\Gamma$ if for any bounded sequence $\left\{x_{n_{j}}\right\}$ with $x_{n_{j}} \in \operatorname{cl}(G) \cap X_{n_{j}}$ such that $P_{n_{j}} T\left(x_{n_{j}}\right) \rightarrow g \in X$, there exists a subsequence $\left\{x_{n_{j(k)}}\right\}$ and an $x \in \operatorname{cl}(G)$ such that $x_{n_{j(k)}} \rightarrow x$ as $k \rightarrow \infty$ and $T(x)=g$.

Such mappings include mappings of the form $I+C$ where $I$ is the identity and $C$ is compact [6, Remark 3, p. 162], mappings of the form $I+S+C$ under certain conditions where $S$ is strictly contractive [6, Theorem 7, p. 162], and $K$-monotone mappings under certain conditions [7, Corollary 2.1, p. 220 and Theorem 2.3, p. 222]. This latter class includes monotone mappings [7, p. 228] and $J$-monotone or accretive mappings [7, pp. 230-231].

The following definition is adapted from Wong [9, p. 406] and makes use of the classical degree in $R^{n}$, deg $(f, D, q)$, called the Brouwer degree of $f$ at $q$ relative to $D$ (cf. [3, Definition 6.3, p. 31] or [8, Definition 3.14, p. 71]). Here $D$ is a bounded open set in oriented Euclidean $n$-space $R^{n}, f$ is a continuous mapping from cl $(D)$ into $R^{n}$, and $q \notin f(\partial D)$. By ${ }^{*} Z$ we denote the ring of all equivalence classes $\left[s_{n}\right]=\left\{\left\{t_{n}\right\}: t_{n}=s_{n}\right.$ for all $n$ sufficiently large $\}$ of sequences of integers.

Definition 3. Let $T:(G) \rightarrow X$ be $A$-proper with respect to a given approximation scheme, where $G_{n}=G \cap X_{n}$ is bounded and open in $X_{n}$ for all $n$ sufficiently large and $T_{n}=\left.P_{n} T\right|_{G_{n}}$ is continuous for all $n$ sufficiently large. Let $y \in X \backslash T(\partial G)$. Then the degree of $T$ at $y$ relative to $G$ is the element $D(T, G, y)=\left[s_{n}\right]$ of ${ }^{*} \mathbf{Z}$ such that

$$
s_{n}=\operatorname{deg}\left(T_{n}, G_{n}, P_{n} y\right)
$$

for all $n$ sufficiently large.

Note that $\operatorname{deg}\left(T_{n}, G_{n}, P_{n} y\right)$ is defined for all $n$ sufficiently large since $P_{n} y \notin T_{n}\left(\partial G_{n}\right)$ for. all $n$ sufficiently large by [2, Lemma 1, p. 220]. The degree of Browder and Petryshyn [1], [2], Deg $(T, G, y)$, is the set of limit points of $\left\{\operatorname{deg}\left(T_{n}, G_{n}, P_{n} y\right)\right\}$ including possibly $\pm \infty$.

In the following the Banach space $l_{2}$ of square summable real sequences with norm $\left\|\left(\alpha_{i}\right)\right\|^{2}=\sum_{i=1}^{\infty} \alpha_{i}^{2}$ will have the oriented projectionally complete scheme $\Gamma\left(l_{2}\right)$ given by

$$
X_{n}=\operatorname{span}\left(e_{1}, e_{2}, \ldots, e_{n}\right) \text { for } n=1,2, \ldots
$$

(where $e_{i}$ has coordinate 1 in the $i$ th place and 0 elsewhere) and

$$
P_{n}\left(\sum_{i=1}^{\infty} \alpha_{i} e_{i}\right)=\sum_{i=1}^{n} \alpha_{i} e_{i} \text { for } n=1,2, \ldots
$$

The orientation of $X_{n}$ is determined by the order $\left(e_{1}, e_{2}, \ldots, e_{n}\right)$ of the basis elements. Let $H_{m}$ be the subset of $l_{2}$ given by

$$
H_{m}=\left\{x \in l_{2}:\left\|m e_{m}-x\right\|<\frac{1}{2}\right\}
$$

and let $G$ be given by

$$
G=\bigcup_{m=1}^{\infty} H_{m}
$$


Then, as is shown in Appendix I, the $H_{m}$ have disjoint closures, cl $\left(H_{m}\right) \cap X_{n}$ is empty for all $m>n$, and $\mathrm{cl}(G)=\cup_{m=1}^{\infty} \mathrm{cl}\left(H_{m}\right)$.

THEOREM. Given any element $\left[s_{n}\right]$ of ${ }^{*} \mathbf{Z}$, there is a mapping $T: \mathrm{cl}(G) \rightarrow l_{2}$ which is A-proper with respect to $\Gamma\left(l_{2}\right)$ and such that $D(T, G, 0)=\left[s_{n}\right]$.

Proof. Let $t_{0}=t_{1}=0$ and $t_{n}=s_{n}$ if $n \geqslant 2$. Then put

$$
k_{n}=\left|t_{n}-t_{n-1}\right|, \quad \varepsilon_{n}=\operatorname{sign}\left(t_{n}-t_{n-1}\right),
$$

and

$$
a_{n}=2 n-1, \quad b_{n}=2 n, \quad n=1,2, \ldots
$$

Define the mapping $T: \mathrm{cl}(G) \rightarrow l_{2}$ as follows. For $x=\sum_{i=1}^{\infty} \alpha_{i} e_{i} \in \operatorname{cl}(G)$ there is a unique $m$ such that $x \in \operatorname{cl}\left(H_{m}\right)$. Then put $T(x)=\sum_{i=1}^{\infty} \eta_{i} e_{i}$ where

$$
\begin{aligned}
\eta_{m-1} & =\varepsilon_{m} \prod_{i=1}^{k_{m}}\left(\alpha_{m}-m-a_{i} \alpha_{m-1}\right), \\
\eta_{m} & =\prod_{i=1}^{k_{m}}\left(\alpha_{m}-m-b_{i} \alpha_{m-1}\right), \\
\eta_{i} & =\alpha_{i} \text { for all } i \neq m-1, m,
\end{aligned}
$$

for $m \geqslant 2$, and $\eta_{i}=\alpha_{i}$ for all $i$ if $m=1$.

First we show $T$ is $A$-proper. Let $\left\{x_{n_{j}} \in G_{n_{j}}\right\}$ be a bounded sequence and $g \in l_{2}$ be such that $P_{n_{j}} T\left(x_{n_{j}}\right) \rightarrow g$. Let

$$
x_{n_{j}}=\sum_{i=1}^{n_{j}} \alpha_{i, n_{j}} e_{i}, \quad P_{n_{j}} T\left(x_{n_{j}}\right)=\sum_{i=1}^{n_{j}} \beta_{i, n_{j}} e_{i}, \quad \text { and } g=\sum_{i=1}^{\infty} \gamma_{i} e_{i} .
$$

Since $\left\{x_{n_{j}}\right\}$ is bounded, there is a $p$ such that $\left\{x_{n_{j}}\right\} \subseteq \cup_{m=1}^{p} \mathrm{cl}\left(H_{m}\right)$, for if $y \in \operatorname{cl}\left(H_{m}\right)$ then $\|y\| \geqslant\left\|m e_{m}\right\|-\left\|y-m e_{m}\right\| \geqslant m-\frac{1}{2}$. There is a subsequence of $\left\{x_{n_{j}}\right\}$ (call it $\left\{x_{n_{j}}\right\}$ again) and a $q \in\{1, \ldots, p\}$ such that $\left\{x_{n_{j}}\right\}$ $\subseteq \mathrm{cl}\left(H_{q}\right)$.

Now $\left\{\alpha_{i, n_{j}}\right\}_{j=1}^{\infty}$ is bounded for each fixed $i$, since $\left|\alpha_{i, n_{j}}\right| \leqslant \frac{1}{2}$ for $i$ $\neq q$ and $\left|\alpha_{q, n_{j}}\right| \leqslant q+\frac{1}{2}$. Hence there is a further subsequence $\left\{x_{n_{j(k)}}\right\}$ of $\left\{x_{n_{j}}\right\}$ and $\alpha_{i}(i=1, \ldots, q)$ such that $\alpha_{i, n_{j(k)}} \rightarrow \alpha_{i}$ as $k \rightarrow \infty, i=1$, $\ldots, q$. Put $\alpha_{i}=\gamma_{i}$ for $i>q$, and $x=\sum_{i=1}^{\infty} \alpha_{i} e_{i}$. Then

$$
\begin{aligned}
\left\|x_{n_{j(k)}}-x\right\| & \leqslant \sum_{i=1}^{q}\left\|\alpha_{i, n_{j(k)}} e_{i}-\alpha_{i} e_{i}\right\|+\left\|\sum_{i=q+1}^{n_{j(k)}} \alpha_{i, n_{j(k)}} e_{i}-\sum_{i=q+1}^{\infty} \alpha_{i} e_{i}\right\| \\
& =\sum_{i=1}^{q}\left|\alpha_{i, n_{j(k)}}-\alpha_{i}\right|+\left\|\sum_{i=q+1}^{\infty}\left(\beta_{i, n_{j(k)}}-\gamma_{i}\right) e_{i}\right\| \\
& \leqslant \sum_{i=1}^{q}\left|\alpha_{i, n_{j(k)}}-\alpha_{i}\right|+\left\|P_{n_{j(k)}} T\left(x_{n_{j(k)}}\right)-g\right\|
\end{aligned}
$$

which tends to zero as $k \rightarrow \infty$. Hence $x_{n_{j(k)}} \rightarrow x$ and $x \in l_{2}$. Indeed $x$ $\in \operatorname{cl}\left(H_{q}\right)$ since $\left\{x_{n_{j(k)}}\right\} \subseteq \operatorname{cl}\left(H_{q}\right)$.

It remains to show that $T(x)=g$. Let $T(x)=\sum_{i=1}^{\infty} \eta_{i} e_{i}$. Assume $q>1$. 
Since $x \in \operatorname{cl}\left(H_{q}\right), \eta_{i}=\alpha_{i}=\gamma_{i}, i \neq q-1, q$,

$$
\begin{aligned}
\eta_{q-1}=\varepsilon_{q} \prod_{i=1}^{k_{q}}\left(\alpha_{q}-q-a_{i} \alpha_{q-1}\right) & =\lim _{k \rightarrow \infty} \varepsilon_{q} \prod_{i=1}^{k_{q}}\left(\alpha_{q, n_{j(k)}}-q-a_{i} \alpha_{q-1, n_{j(k)}}\right) \\
& =\lim _{k \rightarrow \infty} \beta_{q-1, n_{j(k)}}=\gamma_{q-1},
\end{aligned}
$$

and

$$
\begin{aligned}
\eta_{q}=\prod_{i=1}^{k_{q}}\left(\alpha_{q}-q-b_{i} \alpha_{q-1}\right) & =\lim _{k \rightarrow \infty} \prod_{i=1}^{k_{q}}\left(\alpha_{q, n_{j(k)}}-q-b_{i} \alpha_{q-1, n_{j(k)}}\right) \\
& =\lim _{k \rightarrow \infty} \beta_{q, n_{j(k)}}=\gamma_{q} .
\end{aligned}
$$

For the case $q=1$, since $T$ restricted to $\mathrm{cl}\left(H_{1}\right)$ is the identity, $\eta_{i}=\alpha_{i}$ $=\gamma_{i}$ for all $i=1,2, \ldots$ Hence in either case $T(x)=\sum_{i=1}^{\infty} \eta_{i} e_{i}$ $=\sum_{i=1}^{\infty} \gamma_{i} e_{i}=g$, and $T$ is $A$-proper with respect to $\Gamma\left(l_{2}\right)$.

We will now compute the degree of $T$ at 0 relative to $G$. Since $H_{m} \cap X_{n}$ $=\varnothing$ for $m>n$ (see Appendix I), it follows that

$$
G_{n}=G \cap X_{n}=\bigcup_{m=1}^{n} H_{m, n} \text { where } H_{m, n}=H_{m} \cap X_{n} .
$$

Then

$$
\operatorname{deg}\left(T_{n}, G_{n}, 0\right)=\sum_{m=1}^{n} \operatorname{deg}\left(T_{n}, H_{m, n}, 0\right)
$$

by the sum formula for the Brouwer degree (see [3, Theorem 6.8, p. 32] or [8, Theorem 3.16.5, p. 72]) since the $H_{m}$, and thus the $H_{m, n}$, are disjoint for fixed $n$. Now, for $n \geqslant m \geqslant 2, T_{n}$ is the identity on all components except the $(m-1)$ st and $m$ th. Thus, by the reduction formula for the Brouwer degree (see [3, Theorem 10.1 , p. 51] or [8, Theorem 3.16.7, p. 72]),

$$
\operatorname{deg}\left(T_{n}, H_{m, n}, 0\right)=\operatorname{deg}\left(T_{n, m}, H_{m, n} \cap E_{m}, 0\right),
$$

where $E_{m}=\operatorname{span}\left(e_{m-1}, e_{m}\right)$ with orientation induced by the order of the basis elements, and $T_{n, m}$ is equal to $T_{n}$ restricted to $E_{m}$. Let $U_{m}$ be the translation on $E_{m}$ given by

$$
U_{m}(x)=x+m e_{m} .
$$

Then it follows (see Appendix II) that

$$
\operatorname{deg}\left(T_{n, m}, H_{m, n} \cap E_{m}, 0\right)=\operatorname{deg}\left(T_{n, m} U_{m}, U_{m}^{-1}\left(H_{m, n} \cap E_{m}\right), 0\right) .
$$

Now

$$
U_{m}^{-1}\left(H_{m, n} \cap E_{m}\right)=\left\{x \in E_{m}:\|x\|<\frac{1}{2}\right\}
$$

and

$$
T_{n, m} U_{m}\left(\alpha_{m-1} e_{m-1}+\alpha_{m} e_{m}\right)=\gamma_{m-1} e_{m-1}+\gamma_{m} e_{m},
$$


where

$$
\gamma_{m-1}=\varepsilon_{m} \prod_{i=1}^{k_{m}}\left(\alpha_{m}-a_{i} \alpha_{m-1}\right)
$$

and

$$
\gamma_{m}=\prod_{i=1}^{k_{m}}\left(\alpha_{m}-b_{i} \alpha_{m-1}\right)
$$

Hence, as shown by Cronin [3, pp. 38-40],

$$
\operatorname{deg}\left(T_{n, m} U_{m}, U_{m}^{-1}\left(H_{m, n} \cap E_{m}\right), 0\right)=\varepsilon_{m} k_{m} .
$$

Also $T_{n}$ is the identity on $H_{1, n}$ and $0 \notin \mathrm{cl}\left(H_{1, n}\right)$ so deg $\left(T_{n}, H_{1, n}, 0\right)=0$. Hence

$$
\operatorname{deg}\left(T_{n}, G_{n}, 0\right)=\sum_{m=1}^{n} \operatorname{deg}\left(T_{n}, H_{m, n}, 0\right)=\sum_{m=1}^{n} \varepsilon_{m} k_{m}=t_{n}
$$

since $t_{1}=0$. Thus

$$
D(T, G, 0)=\left[t_{n}\right]=\left[s_{n}\right]
$$

\section{Appendix I.}

1. We show that $\mathrm{cl}\left(H_{m}\right) \cap \operatorname{cl}\left(H_{n}\right)=\varnothing$ for $m \neq n$. If $x \in \operatorname{cl}\left(H_{m}\right)$ and $y$ $\in \operatorname{cl}\left(H_{n}\right)$, then

$$
\begin{aligned}
\|x-y\| & \geqslant\left\|m e_{m}-n e_{n}\right\|-\left\|m e_{m}-x\right\|-\left\|n e_{n}-y\right\| \\
& \geqslant\left(m^{2}+n^{2}\right)^{1 / 2}-\frac{1}{2}-\frac{1}{2} \geqslant 2^{1 / 2}-1>0 .
\end{aligned}
$$

Hence $x \neq y$ and $\operatorname{cl}\left(H_{m}\right) \cap \operatorname{cl}\left(H_{n}\right)=\varnothing$.

2. It also follows that $\operatorname{cl}(G)=\operatorname{cl}\left(\cup_{m=1}^{\infty} H_{m}\right)=\cup_{m=1}^{\infty} \mathrm{cl}\left(H_{m}\right)$. For if $\left\{g_{n}\right\} \subseteq G$ is such that $g_{n} \rightarrow x$ then there is an $N$ such that $\left\|g_{n}-g_{m}\right\|$ $\leqslant\left(2^{1 / 2}-1\right) / 2$ for all $n, m \geqslant N$. Hence there is a $p$ such that $g_{n} \in H_{p}$ for all $n \geqslant N$. Hence $x \in \operatorname{cl}\left(H_{p}\right)$ and $\operatorname{cl}(G) \subseteq \cup_{m=1}^{\infty} \operatorname{cl}\left(H_{m}\right)$. Clearly $\cup_{m=1}^{\infty} \operatorname{cl}\left(H_{m}\right) \subseteq \mathrm{cl}(G)$, so $\mathrm{cl}(G)=\cup_{m=1}^{\infty} \mathrm{cl}\left(H_{m}\right)$.

3. We will now show that $\mathrm{cl}\left(H_{m}\right) \cap \operatorname{span}\left(e_{1}, \ldots, e_{n}\right)=\varnothing$ for all $m>n$. If $x \in \operatorname{cl}\left(H_{m}\right)$ and $y=\sum_{i=1}^{n} \alpha_{i} e_{i} \in \operatorname{span}\left(e_{1}, \ldots, e_{n}\right)$ and $m>n$ then

$$
\|x-y\|=\left\|m e_{m}-\sum_{i=1}^{n} \alpha_{i} e_{i}\right\|=\left(m^{2}+\sum_{i=1}^{n} \alpha_{i}^{2}\right)^{1 / 2} \geqslant m>0 .
$$

Hence $x \neq y$ and $\operatorname{cl}\left(H_{m}\right) \cap \operatorname{span}\left(e_{1}, \ldots, e_{n}\right)=\varnothing$, for $m>n$.

Appendix II. Let $D$ be an open bounded subset of $R^{n}, f$ a continuous mapping from cl $(D)$ to $R^{n}$ and $q \notin f(\partial D)$. Let $x_{0} \in X$ be fixed. Let $U$ be the translation defined by $U(x)=x+x_{0}$. We will show that

$$
\operatorname{deg}\left(f U, U^{-1}(D), q\right)=\operatorname{deg}(f, D, q) .
$$

By the product formula for the Brouwer degree [8, Theorem 3.20, p. 75] 


$$
\operatorname{deg}\left(f U, U^{-1}(D), q\right)=\sum_{i} \operatorname{deg}\left(f, B_{i}, q\right) \operatorname{deg}\left(U, U^{-1}(D), p_{i}\right)
$$

where $B_{i}$ are the bounded components of $R^{n} \backslash U\left(\partial U^{-1}(D)\right)=R^{n} \backslash \partial D$ and $p_{i} \in B_{i}$. But

$$
\operatorname{deg}\left(U, U^{-1}(D), p_{i}\right)=\sum \operatorname{sign}\left|U^{\prime}(x)\right|,
$$

where $\left|U^{\prime}(x)\right|$ is the determinant of the Jacobian matrix of $U$ at $x$, and the sum is over all points $x$ in $U^{-1}\left(p_{i}\right) \cap U^{-1}(D)$, i.e. $x=p_{i}-x_{0}, p_{i} \in B_{i} \cap D$. Now $U(x+h)-U(x)=h$ for all $x, h \in R^{n}$, so $U^{\prime}(x)$ is the identity matrix and $\left|U^{\prime}(x)\right|=1$. Hence,

$$
\operatorname{deg}\left(f U, U^{-1}(D), q\right)=\sum_{i} \operatorname{deg}\left(f, B_{i}, q\right),
$$

where the sum is over the bounded components of $D$. Thus by the sum formula for the Brouwer degree (see [3, Theorem 6.8, p. 32] or [8, Theorem 3.16.5, p. 72]), $\operatorname{deg}\left(f U, U^{-1}(D), q\right)=\operatorname{deg}(f, D, q)$.

ACKNOWLDEGEMENT. The author gratefully acknowledges the assistance of Dr. J. J. Koliha in the preparation of this paper.

\section{REFERENCES}

1. F. E. Browder and W. V. Petryshyn, The topological degree and Galerkin approximation for noncompact operators in Banach spaces, Bull. Amer. Math. Soc. 74 (1968), 641-646. MR 37 \#678.

2. Approximation methods and the generalized topological degree for nonlinear mappings in Banach spaces, J. Functional Analysis 3 (1969), 217-245. MR 39 \#6126.

3. J. Cronin, Fixed points and topological degree in nonlinear analysis, Math. Surveys, no. 11, Amer. Math. Soc., Providence, R. I., 1964. MR 29 \# 1400.

4. P. M. Fitzpatrick, $A$ generalized degree for uniform limits of A-proper mappings, J. Math. Anal. Appl. 35 (1971), 536-552. MR 43 \#6788.

5. J. Leray and J. Schauder, Topologie et équations fonctionnelles, Ann. Sci. École Norm. Sup. 51 (1934), 45-78.

6. W. V. Petryshyn, On the approximation solvability of nonlinear equations, Math. Ann. 177 (1968), 156-164. MR 37 \#2048.

7. —_ Nonlinear equations involving noncompact operators, Proc. Sympos. Pure Math., vol. 18, part 1, Amer. Math. Soc., Providence, R. I., 1970, pp. 206-233. MR 42 \#6670.

8. J. T. Schwartz, Non-linear functional analysis, Gordon and Breach, New York, 1969.

9. H. S. F. Wong, The topological degree of A-proper maps, Canad. J. Math. 23 (1971), 403-412. MR 44 \#5843.

10. 361-371. MR 49 \# 9867.

Department of Mathematics, University of Melbourne, Parkville, Victoria 3052, Australia 